\title{
LOCAL MAPPING RELATIONS AND GLOBAL IMPLICIT FUNCTION THEOREMS $\left({ }^{1}\right)$
}

\author{
BY \\ WERNER C. RHEINBOLDT \\ Dedicated to Professor H. Goertler on his sixtieth birthday
}

1. Introduction. Consider the problem of solving a nonlinear equation

$$
F(x, y)=z
$$

with respect to $y$ for given $x$ and $z$. If, for example, $x, y, z$ are elements of some Banach space, then under appropriate conditions about $F$, the well-known implicit function theorem ensures the "local" solvability of (1). The question arises when such a local result leads to "global" existence theorems. Several authors, for example, Cesari [2], Ehrmann [5], Hildebrandt and Graves [8], and Levy [9], have obtained results along this line. But these results are closely tied to the classical implicit function theorem, and no general theory appears to exist which permits the deduction of global solvability results once a local one is known.

The problem has considerable interest in numerical analysis. In fact, when an operator equation

$$
F y=x
$$

is to be solved iteratively for $y$, the process converges usually only in a neighborhood of a solution. If for some other operator $F_{0}$ the equation $F_{0} y=x$ has been solved, then one may try to "connect" $F$ and $F_{0}$ by an operator homotopy $H(t, y)$ such that $H(0, y)=F_{0} y$ and $H(1, y)=F y$, and to "move" along the solution "curve" $y(t)$ of $H(t, y)=x, 0 \leqq t \leqq 1$, from the known solution $y(0)$ to the unknown $y(1)$, for instance, by using a locally convergent iterative process.

This is the so-called "continuation method". For a review of earlier work about this method see, for example, the introduction of Ficken [6] who then proceeds to develop certain results about the global solvability of $H(t, y)=x$ by using the local solvability provided by the implicit function theorem. Other results are due to Davidenko [3]; see also Yakovlev [14] and, more recently, Davis [4], and Meyer [10].

In this paper we shall consider the problem of finding global existence theorems for (1) in the following setting. For fixed $z$ the problem depends only on the (multivalued) relation between $x$ and $y$ given by (1). We therefore consider abstract

Received by the editors March 21, 1968.

(1) This work was in part supported by the National Aeronautics and Space Administration under Grant NsG 398 and by the U.S. Army Research Office (Durham) under Grant DA-AROD31-124-G676. 
relations $\phi$ between elements of a topological space $X$ and another such space $Y$. The assumed existence of a local solution of (1) leads to the condition that $\phi$ behaves locally like a continuous mapping. The problem then is to obtain from this "local knowledge" information about the global behavior of $\phi$. The central concept is a general "continuation property" which in turn is equivalent to a so-called "path-lifting property". The latter states that for a continuous path $p$ in $X$ a path $q$ in $Y$ can be found such that $p$ and $q$ are point-wise related under $\phi$. The resulting theory resembles (and was in part influenced by) the theory of covering mappings in algebraic topology.

The theoretical results of $\S 2$ are applied in $\S 3$ to the case of equation (2), and in $\S 4$ to that of (1). The results of $\$ 3$ contain some new global existence theorems for (2) and also include the well-known Hadamard-Levy theorem (see [7] and [9]) as well as a recent generalization by Meyer [10]. $\$ 4$ essentially covers and extends the results of Ficken [6].

Although some of the specific applications in $\$ \S 3$ and 4 use the implicit function theorem, the main results do not depend on the way the local invertibility is ensured, and hence corresponding results based on other local solvability theorems can conceivably be phrased.

At this point, I would like to express my thanks to Professor James M. Ortega of the University of Maryland for his critical comments and many helpful discussions during the preparation of this paper.

2. Local mapping relations. Let $X, Y$ be Hausdorff topological spaces. We consider relations $\phi \subset X \times Y$ with domain $D(\phi)$ in $X$ and range $R(\phi)$ in $Y$, and we shall use the notations $(x, y) \in \phi$ and $x \phi y$ interchangeably. Moreover, for $Q \subset X$ we write $\phi[Q]=\{y \in Y \mid x \phi y$ for some $x \in Q \cap D(\phi)\}$. The inverse of $\phi$ is as usual denoted by $\phi^{-1}$.

The following definition introduces the local solvability property mentioned in the introduction.

2.1. Definition. A relation $\phi \subset X \times Y$ is said to be a local mapping relation, or to have the local mapping property, if for each $\left(x_{0}, y_{0}\right) \in \phi$ there exist relatively open neighborhoods $U\left(x_{0}\right)$ of $x_{0}$ in $D(\phi)$ and $V\left(y_{0}\right)$ of $y_{0}$ in $R(\phi)$, such that the restriction $\varphi=\phi \cap\left(U\left(x_{0}\right) \times V\left(y_{0}\right)\right)$ is a continuous mapping from $U\left(x_{0}\right)$ into $V\left(y_{0}\right)$.

For the sake of brevity we call $U\left(x_{0}\right), V\left(y_{0}\right)$ a pair of canonical neighborhoods of $\left(x_{0}, y_{0}\right) \in \phi$ and $\varphi: U\left(x_{0}\right) \rightarrow V\left(y_{0}\right)$ the corresponding canonical mapping. For reasons of simplicity we shall frequently assume that $D(\phi)=X$.

If $F: Y \rightarrow X$ is a continuous map from $Y$ into $X$, then the graph of $F$,

$$
\Gamma_{F}=\{(x, y) \in Y \times X \mid x=F y\},
$$

is clearly a local mapping relation in $Y \times X$ with $D(\phi)=F(Y)$. More interesting is the question when the inverse graph $\Gamma_{F}^{-1}$ is a local mapping relation, that is, when $F$ has locally a continuous inverse. This will be discussed in $\S 3$. The case of the relation between $x$ and $y$ defined by (1) ( $z$ fixed) will be the topic of $\S 4$. 
Given $Q \subset X$, we denote by $\mathscr{P}(Q)$ the set of all possible continuous paths $p:[0,1]=J \subset R^{1} \rightarrow Q$ in $Q$. As usual, two paths $p, q \in \mathscr{P}(Q)$ are called equal, in symbols $p \cong q$, if $p(\tau(t))=q(t), t \in J$, where $\tau: J \rightarrow J, \tau(0)=0, \tau(1)=1$ is a continuous, strongly isotone "parameter transformation".

Generally, we are concerned with the question when the existence of local solutions guaranteed by the local mapping property assures the existence of "global" solutions. The tool for studying this problem will be the following "continuation" concept. Given a local mapping relation $\phi \subset X \times Y$ with $D(\phi)=X$ and some path $p \in \mathscr{P}(X)$, we are interested in finding "solutions" $y \in Y$ of $p(t) \phi y, t \in J=[0,1]$. Suppose that $y_{0} \in Y$ is known with $p(0) \phi y_{0}$ and that $\varphi$ is the canonical mapping corresponding to $\left(p(0), y_{0}\right)$. Then $q(t)=\varphi(p(t))$ is uniquely and continuously defined for some interval $0 \leqq t<\varepsilon$. Since $q(0)=y_{0}$, we can call $q$ a "continuation" of the initial solution $y_{0}$ of the problem $p(t) \phi y, t \in J$ for small values of $t$. If $q$ is still defined for $t_{0}>0$, then the process can be repeated with $y_{1}=q\left(t_{1}\right)$ in place of $y_{0}$. The question arises whether it is possible to "continue" the solution for all $t$ in $J$, that is, whether there exists a path $q \in \mathscr{P}(Y)$ such that $p(t) \phi q(t)$ for $t \in J$ and $q(0)=y_{0}$. Without additional assumptions about $\phi$, this is, of course, not the case. But it turns out that when a path $q$ of the described type does exist for certain paths $p$ and for all corresponding initial solutions, then a number of interesting results can be proved about the global behavior of $\phi$. Accordingly, we introduce the following condition:

2.2. Definition. A relation $\phi \subset X \times Y$ is said to have the continuation property for the subset $\mathscr{P}_{X} \subset \mathscr{P}(X)$ if for any $p \in \mathscr{P}_{X}$ and any continuous function $q:[0, \hat{t}) \subset J \rightarrow Y$ with $p(t) \phi q(t)$ for $t \in[0, \hat{t})$ there exists a sequence $\left\{t_{k}\right\} \subset[0, \hat{t})$ with $\lim _{k \rightarrow \infty} t_{k}=\hat{t}$ such that $\lim _{k \rightarrow \infty} q\left(t_{k}\right)=\hat{y}$ and $p(\hat{t}) \phi \hat{y}$.

Before showing that this condition indeed allows the continuation process to be carried out until $t=1$, it is useful to introduce the following terminology:

2.3. Definition. A relation $\phi \subset X \times Y$ is said to have the path-lifting property for a set $\mathscr{P}_{X} \subset \mathscr{P}(X)$ if for any $p \in \mathscr{P}_{X}$ and any $y_{0} \in \phi[p(0)]$ there exists a path $q \in \mathscr{P}(Y)$ such that $q(0)=y_{0}$ and $p(t) \phi q(\tau(t))$ for $t \in J$ and some parameter transformation $\tau$. We call $q$ a lifting of $p$ through $y_{0}$ and write $p \phi q$. The set of all paths in $\mathscr{P}(Y)$ which occur as liftings of paths of $\mathscr{P}_{X}$ will be denoted by $\phi\left[\mathscr{P}_{X}\right]$.

This definition represents a simple modification of the lifting concept used in the theory of covering maps in algebraic topology. The announced result can now be phrased as follows:

2.4. TheOREM. Let $\phi \subset X \times Y$ be a local mapping relation with $D(\phi)=X$. Then $\phi$ has the path-lifting property for $\mathscr{P}_{X} \subset \mathscr{P}(X)$ if and only if $\phi$ has the continuation property for $\mathscr{P}_{X}$.

Proof. The necessity of the statement is obvious. Suppose therefore that $\phi$ has the continuation property for $\mathscr{P}_{X}$. If $p \in \mathscr{P}_{X}$ and $y_{0} \in Y$ with $p(0) \phi y_{0}$ are given, the continuation process ensures the existence of a continuous $q:[0, \hat{t}) \subset J \rightarrow Y$ 
with $q(0)=y_{0}, p(t) \phi q(t)$ for $t \in[0, \hat{t})$ and some $\hat{t} \in(0,1]$. Let $\hat{t}$ be the maximal value with this property in $J$. By assumption, a sequence $\left\{t_{k}\right\} \subset[0, \hat{t})$ exists with $\lim _{k \rightarrow \infty} t_{k}$ $=\hat{t}, \lim _{k \rightarrow \infty} q\left(t_{k}\right)=\hat{y}$ and $p(\hat{t}) \phi \hat{y}$, and, using the local mapping property, it follows readily that by setting $q(\hat{t})=\hat{y}, q$ is continuously defined for $0 \leqq t \leqq \hat{t}$. If $\hat{t}<1$, then $q$ can be continued beyond $\hat{t}$ which contradicts the maximality of $\hat{t}$. Therefore, $\hat{t}=1$ and $\phi$ has the path-lifting property for $\mathscr{P}_{X}$.

We turn now to the consequences of the path-lifting property. A first result states that a lifting of a path $q$ is uniquely determined by the initial point $y_{0}$. This fact is hardly surprising in view of the continuation idea behind the path-lifting concept.

2.5. THEOREM. Let $\phi \subset X \times Y, D(\phi)=X$, be a local mapping relation with the path-lifting property for $\mathscr{P}_{X} \subset \mathscr{P}(X)$. If $q_{1}, q_{2} \in \mathscr{P}(Y)$ are two liftings of $p \in \mathscr{P}_{X}$ with $q_{1}(0)=q_{2}(0)=y_{0}$, then $q_{1} \cong q_{2}$.

Proof. By assumption we have-possibly after suitable parameter transformations- $p(t) \phi q_{i}(t), \quad t \in J, \quad i=1,2$. Then $0 \in J_{0}=\left\{t \in J \mid q_{1}(s)=q_{2}(s), \quad s \in[0, t]\right\}$ implies that $\hat{t}=\sup \left\{t \mid t \in J_{0}\right\}$ is well defined, and by continuity we obtain $\hat{t} \in J_{0}$. If $\hat{t}<1$ then by the local mapping property $q_{1}(t)=\varphi(p(t))=q_{2}(t)$ for a small interval around $\hat{t}$ where $\varphi$ is the canonical map at $\left(p(\hat{t}), q_{1}(\hat{t})\right) \in \phi$. This contradicts the construction of $\hat{t}$ and hence we have $\hat{t}=1$ and therefore $q_{1} \cong q_{2}$.

For the further discussion we have to restrict the class of permissible subsets $\mathscr{P}_{X}$ of $\mathscr{P}(X)$. Given $\mathscr{P}_{X} \subset \mathscr{P}(X)$, a set $Q \subset X$ is said to be $\mathscr{P}_{X}$-path connected if any two points $x_{1}, x_{2} \in Q$ are connected by a path from $\mathscr{P}_{X} \cap \mathscr{P}(Q)$. Analogously, the usual concept of local $\mathscr{P}_{X}$-path connectedness, and of $\mathscr{P}_{X}$-path components of $Q$ are introduced. If $\mathscr{P}_{X}$ is a so-called admissible subset of $\mathscr{P}(X)$, that is, if $\mathscr{P}_{X}$ is closed under path-reversal, path-segmentation, and finite concatenation of paths, then $Q$ can be partitioned into disjoint $\mathscr{P}_{X}$-path components.

Note that when $\phi \subset X \times Y$ is a local mapping relation with the path-lifting property for the admissible set $\mathscr{P}_{X} \subset \mathscr{P}(X)$ then $\phi\left[\mathscr{P}_{X}\right]$ is evidently also admissible.

As an application of 2.5 we obtain now the following result:

2.6. ThEOREM. Let $\phi \subset X \times Y, D(\phi)=X$, be a local mapping relation with the path-lifting property for the admissible set $\mathscr{P}_{X} \subset \mathscr{P}(X)$. Assume further that $X$ is locally $\mathscr{P}_{X}$-path connected and let $X=\bigcup_{\mu \in M} X_{\mu}$ be the decomposition of $X$ into its $\mathscr{P}_{X}$-path components. Then, each one of the partial relations $\phi_{\mu}=\phi \cap\left(X_{\mu} \times Y\right)$ is a local mapping relation with the path-lifting property for $\mathscr{P}_{\mu}=\mathscr{P}_{X} \cap \mathscr{P}\left(X_{\mu}\right)$. Moreover, for fixed $\mu$ the cardinality of the set $\phi_{\mu}[x]$ is independent of the choice of $x \in X_{\mu}$.

Proof. If $\left(x_{0}, y_{0}\right) \in \phi_{\mu}$ and $x \in X_{\mu}$, then there exists a $p \in \mathscr{P}_{\mu}$ connecting $x_{0}$ and $x$ and hence also a path $q \in \mathscr{P}(Y)$ with $q(0)=y_{0}$ and $p \phi q$. Thus, $x \phi q(1)$ and therefore $x \in D\left(\phi_{\mu}\right)$. Let $U\left(x_{0}\right), V\left(y_{0}\right)$ be canonical neighborhoods under $\phi$ and $\varphi$ the canonical map. Then there is an open $\mathscr{P}_{X}$-path-connected neighborhood $U_{0}\left(x_{0}\right)$ $\subset U\left(x_{0}\right)$ of $x_{0}$ in $X$, and clearly $U_{0}\left(x_{0}\right), V\left(y_{0}\right)$ constitutes a pair of canonical neigh- 
borhoods of $\left(x_{0}, y_{0}\right) \in \phi_{\mu}$ with the restriction $\varphi \mid U_{0}\left(x_{0}\right)$ of $\varphi$ as corresponding canonical map. Hence, $\phi_{\mu}$ is again a local mapping relation and evidently $\phi_{\mu}$ inherits from $\phi$ the path-lifting property.

For the proof of the last part let $x_{0}, x_{1} \in X_{\mu}$; then there is a path $p \in \mathscr{P}_{\mu}$ connecting them and for each $y_{0} \in \phi_{\mu}\left[x_{0}\right]$ there exists a unique lifting $q \in \mathscr{P}(Y)$ of $p$ through $y_{0}$. Thus, $q(1) \in \phi_{\mu}\left[x_{1}\right]$ and by 2.5 the correspondence $y_{0} \rightarrow q(1)$ is for fixed $p$ a mapping $\pi$ from $\phi_{\mu}\left[x_{0}\right]$ into $\phi_{\mu}\left[x_{1}\right]$. This mapping is injective; in fact, if $q_{i} \in \mathscr{P}(Y), i=1,2$ are liftings of $p$ with $q_{1}(1)=q_{2}(1)$, then the reverse paths $\bar{q}_{i}$ are liftings of the reverse path $\bar{p}$ and hence 2.5 implies that $\bar{q}_{1} \cong \bar{q}_{2}$ and therefore $q_{1}(0)=q_{2}(0)$. If the reverse path $\bar{p}$ is used to define in the same manner a mapping $\bar{\pi}$ from $\phi_{\mu}\left[x_{1}\right]$ into $\phi_{\mu}\left[x_{0}\right]$, it follows again from 2.5 that $\bar{\pi}=\pi^{-1}$, and thus $\pi$ is bijective. Therefore, $\phi_{\mu}\left[x_{0}\right]$ and $\phi_{\mu}\left[x_{1}\right]$ have the same cardinality.

For $\mathscr{P}_{X} \subset \mathscr{P}(X)$, a $\mathscr{P}_{X}$-path homotopy on $Q \subset X$ is defined as a continuous map $g: J \times J \subset R^{2} \rightarrow Q$ such that for fixed $s_{0}, t_{0} \in J, g\left(s_{0}, \cdot\right)$ and $g\left(\cdot, t_{0}\right)$ are paths of $\mathscr{P}_{X}$. Equality of $\mathscr{P}_{X}$-path homotopies in $Q$, in symbols $g_{1} \cong g_{2}$, is defined by $g_{1}\left(\tau_{1}(s), \tau_{2}(t)\right)=g_{2}(s, t), s, t \in J$, where $\tau_{1}$ and $\tau_{2}$ are parameter transformations.

As in the theory of covering mappings, it follows now that local mapping relations with the path-lifting property for $\mathscr{P}_{X}$ also lift $\mathscr{P}_{X}$-path homotopies. In fact, the proof of the following theorem is an adaptation of a corresponding proof of Schubert [12] for covering maps.

2.7. ThEOREM. Let $\phi \subset X \times Y, D(\phi)=X$, be a local mapping relation with the path-lifting property for $\mathscr{P}_{X} \subset \mathscr{P}(X)$. For a $\mathscr{P}_{X}$-path homotopy $g$ in $X$ and a path $q^{0} \in \mathscr{P}(Y)$ with $g(\cdot, 0) \phi q^{0}$ there exists a $\mathscr{P}(Y)$-path homotopy $h$ in $Y$ and parameter transformations $\tau_{1}, \tau_{2}$ such that $g(s, t) \phi h\left(\tau_{1}(s), \tau_{2}(t)\right), s, t \in J$, and $h(\cdot, 0) \cong q^{0}$. Moreover, if $\hat{h}$ is any other $\mathscr{P}(Y)$-path homotopy in $Y$ with the same properties, then $h \cong \hat{h}$.

Proof. For fixed $s \in J, p_{s}(t)=g(s, t), t \in J$ defines a path of $\mathscr{P}_{X}$ and hence there exists a unique path $q_{s} \in \mathscr{P}(Y)$ with $p_{s} \phi q_{s}$ and $q_{s}(0)=q^{0}(s)$. Let $h$ be any $\mathscr{P}(Y)$-path homotopy in $Y$ with the stated properties. Then, after a suitable parameter transformation, we have $h(s, 0)=q^{0}(s)$ for any $s \in J$ and hence by $2.5, h(s, \cdot) \cong q_{s}, s \in J$. This shows that $h$ is uniquely determined up to parameter transformations. Therefore, if $h: J \times J \subset R^{2} \rightarrow Y$ is defined by $h(s, t)=q_{s}(t), s, t \in J$, then all that remains is to prove the continuity of $h$ on $J \times J$.

Suppose $g$ is discontinuous at $\left(s_{0}, t_{1}\right) \in J \times J$ and that $t_{0}$ is the infimum of all those $t \in J$ for which $g$ is discontinuous at $\left(s_{0}, t\right)$. Set $x_{0}=g\left(s_{0}, t_{0}\right)$ and $y_{0}=h\left(s_{0}, t_{0}\right)$, and let $U\left(x_{0}\right), V\left(y_{0}\right)$ be a pair of canonical neighborhoods and $\varphi$ the corresponding canonical map. Since $h$ is continuous on $J \times J$, there exist open neighborhoods $J\left(s_{0}\right)$ and $J\left(t_{0}\right)$ of $s_{0}$ and $t_{0}$ in $J$ such that $g\left(J\left(s_{0}\right), J\left(t_{0}\right)\right) \subset U\left(x_{0}\right)$. We distinguish now two cases:

CASE 1. $t_{0}=0$. Then $y_{0}=h\left(s_{0}, 0\right)=q^{0}\left(s_{0}\right)$ and because of the continuity of $q^{0}$ there exists an open neighborhood $J^{\prime}\left(s_{0}\right)$ of $s_{0}$ in $J$ such that $q^{0}\left(J^{\prime}\left(s_{0}\right)\right) \subset V\left(x_{0}\right)$. It 
is no restriction to assume that $J^{\prime}\left(s_{0}\right)=J\left(s_{0}\right)$. This means that $\varphi(g(s, 0))=q^{0}(s)$ $=h(s, 0)$ for $(s, 0) \in J\left(s_{0}\right) \times J(0)$ and therefore $\varphi(g(s, t))=\varphi\left(p_{s}(t)\right)=q_{s}(t)=h(s, t)$ for $(s, t) \in J\left(s_{0}\right) \times J(0)$. With $g$ also $h$ is continuous at all points of $J\left(s_{0}\right) \times J(0)$ and this contradicts the construction of $t_{0}=0$.

CASE 2. $t_{0}>0$. Since $p_{s_{0}}(\cdot)$ is continuous in $t$, we can choose $t^{\prime}<t_{0}$ such that $t^{\prime} \in J\left(t_{0}\right)$ and that $h\left(s_{0}, t^{\prime}\right)=q_{s_{0}}\left(t^{\prime}\right) \in V\left(y_{0}\right)$. By assumption $h$ is continuous in both variables at $\left(s_{0}, t^{\prime}\right)$ and hence there exists a neighborhood $J^{\prime}\left(s_{0}\right)$ of $s_{0}$ in $J$, which we can assume to be equal to $J\left(s_{0}\right)$, such that $h\left(J\left(s_{0}\right), t^{\prime}\right) \subset V\left(y_{0}\right)$. But then 2.5 implies again that $\varphi(g(s, t))=\varphi\left(p_{s}(t)\right)=q_{s}(t)=h(s, t)$ for each $s \in J\left(s_{0}\right)$ and all $t \in J\left(t_{0}\right)$. This means that $h$ is continuous in an entire neighborhood of $\left(s_{0}, t_{0}\right)$ in contradiction to the construction of $t_{0}$. This completes the proof.

This theorem permits us to prove the following "global" result for local mapping relations. We call the space $X \mathscr{P}_{X}$-simply-connected if it is path-connected under $\mathscr{P}_{X}$ and if any two paths of $\mathscr{P}_{X}$ with the same endpoints are $\mathscr{P}_{X}$-homotopic.

2.8. THEOREM. Let $\phi \subset X \times Y, D(\phi)=X$, be a local mapping relation with the path-lifting property for the admissible set $\mathscr{P}_{X} \subset \mathscr{P}(X)$ and suppose that $X$ is $\mathscr{P}_{X^{-}}$ simply-connected and locally $\mathscr{P}_{X}$-path-connected. Then there exists a family of continuous mappings $F_{\mu}: X \rightarrow R(\phi)$ such that $x \phi F_{\mu} x$ and $\phi[x]=\left\{F_{\mu} x\right\}_{\mu \in M}$, where $M$ is the fixed index set introduced in 2.6. Moreover, each set $Q_{\mu}=F_{\mu}(X), \mu \in M$, is $\phi\left[\mathscr{P}_{\mathrm{X}}\right]$-path-connected.

Proof. For given $x_{0}, x \in X$ we generated in the proof of 2.6 a bijection $\pi$ between $\phi\left[x_{0}\right]$ and $\phi[x]$ as follows: Let $p$ be a fixed path connecting $x_{0}$ and $x$ and $y_{0} \in \phi\left[x_{0}\right]$ any point; then there exists a unique lifting $q$ of $p$ with initial point $y_{0}$, and $\pi$ is defined by $\pi\left(y_{0}\right)=q(1)$. If $X$ is $\mathscr{P}_{X}$-simply-connected, then for given $x_{0}, x \in X$ the bijection $\pi=\pi_{x_{0} x}$ from $\phi\left[x_{0}\right]$ onto $\phi[x]$ does not depend on the choice of $p$. To see this, let $p^{\prime}$ be any other path connecting $x_{0}$ with $x$ and $q^{\prime}$ the lifting of $p^{\prime}$ with initial point $y_{0} \in \phi\left[x_{0}\right]$. There exists a homotopy $g$ in $X$ with $g(0, t)=p(t), g(1, t)=p^{\prime}(t)$, $(t \in J), g(s, 0)=x_{0}, g(s, 1)=x,(s \in J)$. Hence, 2.7 implies the existence of a unique path homotopy $h$ in $Y$ for which-after a suitable parameter transformation$g(s, t) \phi h(s, t)$ and $h(s, 0)=y_{0},(s, t \in J)$. Therefore, it follows from 2.5 that $h(0, \cdot)$ $\cong q, h(1, \cdot) \cong q^{\prime}$ and hence that $y=q(1)=h(0,1)$ and $y^{\prime}=q^{\prime}(1)=h(1,1)$. But then, $x=g(s, 1) \phi h(s, 1)$ for $s \in J$, together with the continuity of $h(\cdot, 1)$, and the local mapping property of $\phi$ necessarily implies that $y=y^{\prime}$.

Now let $x_{0} \in X$ be a fixed point and $\phi\left[x_{0}\right]=\left\{y_{\mu}\right\}_{\mu \in M}$. By 2.6 the cardinality of the index set $M$ is invariant under changes of $x_{0}$. For given $y_{\mu} \in \phi\left[x_{0}\right]$ there exists for each $x \in X$ a unique $y=\pi\left(y_{\mu}\right) \in \phi[x]$ where $\pi=\pi_{x_{0} x}$ is the bijection constructed above. Let $Q_{\mu}=\left\{y \in R(\phi) \mid y=\pi\left(y_{\mu}\right)\right\}$; then, because of the bijectivity of $\pi$, it follows immediately that every $y \in R(\phi)$ must be contained in at least one $Q_{\mu}$, i.e., that $\bigcup_{\mu \in M} Q_{\mu}=R(\phi)$. Clearly, each $Q_{\mu}$ is $\phi\left[\mathscr{P}_{X}\right]$-path connected.

Evidently, the correspondence $x \in X \rightarrow y=\pi_{x_{0} x}\left(y_{\mu}\right) \in Q_{\mu}$ defines a mapping $F_{\mu}: X \rightarrow R(\phi)$ with $F_{\mu}(X)=Q_{\mu}$. In view of the properties of $\pi_{x_{0} x}$ we then have 
$x \phi F_{\mu} x$ and $\phi[x]=\left\{\pi_{x_{0} x}\left(y_{\mu}\right)\right\}_{\mu \in M}$. It remains to be shown that $F_{\mu}$ is continuous as a mapping from $X$ into $R(\phi)$. For this we prove that locally $F_{\mu}$ coincides with the canonical mappings. For given $\hat{x} \in X, \hat{y}=F_{\mu} \hat{x}$, let $U(\hat{x}), V(\hat{y})$ be a pair of canonical neighborhoods with the canonical map $\varphi$. Since $X$ is locally $\mathscr{P}_{X}$-path connected, it is no restriction to assume that $U(x)$ is $\mathscr{P}_{x}$-path connected. Let $x \in U(\hat{x})$ and $y=\varphi(x) \in V(\hat{y})$, then there exists a $p^{\prime \prime} \in \mathscr{P}_{X}$ from $\hat{x}$ to $x$ in $U(\hat{x})$ and hence, if $p^{\prime}$ is any path from $x_{0}$ to $\hat{x}$, the concatenation $\left(p^{\prime} p^{\prime \prime}\right)^{-}$defines a path $p$ from $x_{0}$ to $x$. Now let $q^{\prime}$ be any lifting of $p^{\prime}$ with initial point $y_{\mu}$, then $q^{\prime}(1)=F_{\mu} \hat{x}=\hat{y}$ and, since $q^{\prime \prime}(t)=\varphi\left(p^{\prime \prime}(t)\right)(t \in J)$ defines a lifting of $p^{\prime \prime}$ with initial point $\hat{y}$, it follows that the concatenation $\left(q^{\prime} q^{\prime \prime}\right)^{-}$provides us with a lifting $q$ of $p$ with initial point $y_{\mu}$. But then, by definition of $F_{\mu}$, we have $F_{\mu} x=q(1)=y=\varphi(x)$ as stated. The continuity of $F_{\mu}$ is now an easy consequence.

Note that in general the $Q_{\mu}$ are not disjoint as the simple example shows: $X=[0,2] \subset R^{1}, \quad Y=[0,3] \subset R^{1}$ and $\phi[x]=\{2-x, 3-x\}, 0 \leqq x \leqq 2$. Here we have $M=\{1,2\}, Q_{1}=[0,2], Q_{2}=[1,3]$ and $F_{1} x=2-x, F_{2} x=3-x$.

An important case when the $Q_{\mu}$ are disjoint and in fact when they are the $\phi\left[\mathscr{P}_{X}\right]$-path components of $Y$ will be discussed in the next section.

We conclude this section with two somewhat different results. The first provides a sufficient condition for a relation to be a local mapping relation with the pathlifting property, and the second uses the path-lifting property to determine the domain of a local mapping relation.

The following definition represents an extension of the definition of a covering map; accordingly, a corresponding name is used.

2.9. Definition. A relation $\phi \subset X \times Y$ with $D(\phi)=X$ is called a covering relation if for each $x \in X$ there exists an open neighborhood $U(x)$ such that $\phi[U(x)]=\bigcup_{\mu \in M} V_{\mu}$ where the $V_{\mu}$ are disjoint (relatively) open sets in $R(\phi)$ and for each $\mu \in M$ the restriction $\varphi_{\mu}=\phi \cap\left(U(x) \times V_{\mu}\right)$ is a continuous mapping from $U(x)$ into $V_{\mu} . U(x)$ is called an admissible neighborhood of $x$.

2.10. THEOREM. A covering relation $\phi \subset X \times Y$ is a local mapping relation with the path-lifting property for $\mathscr{P}(X)$.

Proof. Let $\left(x_{0}, y_{0}\right) \in \phi, U\left(x_{0}\right)$ an admissible neighborhood of $x_{0}$ and $\phi\left[U\left(x_{0}\right)\right]$ $=\bigcup_{\mu \in M} V_{\mu}$, where the $V_{\mu}$ are as stated in 2.9. Then, $y_{0} \in V_{\mu_{0}}$ for exactly one $\mu_{0} \in M$, and $U\left(y_{0}\right), V_{\mu}$ is evidently a pair of canonical neighborhoods of $\left(x_{0}, y_{0}\right) \in \phi$, i.e., $\phi$ is a local mapping relation.

Let now $p \in \mathscr{P}(X)$ be any path. Since $p(J)$ is compact, there exists a partition $0=t_{0}<t_{1}<\cdots<t_{k+1}=1$ such that $p(t) \in U_{i}$ for $t_{i} \leqq t \leqq t_{i+1}$ where $U_{i}=U\left(p\left(t_{i}\right)\right)$, $i=0,1, \ldots, k$ are admissible neighborhoods of $p\left(t_{i}\right)$. For given $y_{0} \in \phi[p(0)]$ we use induction to construct the lifting of $q$ of $p$ through $y_{0}$. Suppose $q$ has already been defined for $0 \leqq t \leqq t_{i}, i \geqq 0$. Then, $q\left(t_{i}\right) \in \phi\left[p\left(t_{i}\right)\right] \subset \phi\left[U_{i}\right]=\bigcup_{\mu \in M} V_{\mu}$ and $q\left(t_{i}\right)$ belongs to exactly one $V_{\mu_{0}}$. Since $\varphi_{\mu}=\phi \cap\left(U_{i} \times V_{\mu_{0}}\right)$ is a continuous map from $U_{i}$ into $V_{\mu_{0}}$ and since $p(t) \in U_{i}$ for $t_{i} \leqq t \leqq t_{i+1}$, it follows that $q(t)=\varphi_{\mu}(p(t))\left(t_{i} \leqq t \leqq i_{i+1}\right)$ 
represents a well-defined continuous extension of $q$, i.e., $q$ is now defined for $0 \leqq t \leqq t_{i+1}$. This completes the induction, and, therefore, $\phi$ has the path-lifting property.

2.11. THEOREM. Let $\phi \subset X \times Y$ be a local mapping relation, where $D(\phi)$ is open in $X$, and $X$ is $\mathscr{P}_{X}$-path-connected for a set $\mathscr{P}_{X} \subset \mathscr{P}(X)$ which is closed under segmentation. Suppose further that $\phi$ has the continuation property for $\mathscr{P}_{X}$, then $D(\phi)=X$.

Proof. Let $\left(x_{0}, y_{0}\right) \in \phi, x \in X$, and $p \in \mathscr{P}_{X}$ be a path between $x_{0}$ and $x$. Then, $J_{0}=\{t \in J \mid p(t) \in D(\phi)\}$ is not empty and since $D(\phi)$ is open in $X, J_{0}$ is open in $J$. If $J_{0}$ is a proper subset of $J$, there exists a $\hat{t} \in J$ such that $[0, \hat{t}) \subset J_{0}$ but $\hat{t} \notin J_{0}$. For any $t^{\prime} \in[0, \hat{t})$ the closed segment of $p$ from $p(0)$ to $p\left(t^{\prime}\right)$ is a path $p^{\prime}$ in $\mathscr{P}_{X}$ and hence $p^{\prime}$ can be lifted into $Y$. Since $t^{\prime}$ was arbitrary, this implies the existence of a continuous function $q:[0, \hat{t}) \subset J \rightarrow Y$ with $p(t) \phi q(t)$ for $0 \leqq t<\hat{t}$. Therefore, by the continuation property, there exists a sequence $\left\{t_{k}\right\} \subset[0, \hat{t})$ such that $\lim _{k \rightarrow \infty} t_{k}=\hat{t}$, $\lim _{k \rightarrow \infty} q\left(t_{k}\right)=\hat{y}$ and $p(\hat{t}) \phi \hat{y}$. But this implies that $\hat{t} \in J_{0}$, against assumption, and hence we have $J_{0}=J$, and therefore $x \in D(\phi)$, i.e., $D(\phi)=X$.

3. Applications to equations $F y=x$. Throughout this section we consider a map $F: Y \rightarrow X$ from $Y$ into $X$ and the corresponding inverse graph

$$
\phi=\Gamma_{F}^{-1}=\{(x, y) \in X \times Y \mid x=F y\} .
$$

The following theorem concerns the question when $\phi$ is a local mapping relation.

3.1. Theorem. Given a mapping $F: Y \rightarrow X$ from $Y$ into $X$, the inverse graph $\Gamma_{F}^{-1}$ is a local mapping relation if and only if $F$ is open and locally one-to-one.

The proof is an almost immediate consequence of the definitions and is omitted here.

For the following we shall assume that $F$ is a local homeomorphism in the usual sense, i.e., that for each $y \in Y$ there exist open neighborhoods $U(F y)$ of $F y$ in $F(Y)$ and $V(y)$ of $y$ in $Y$, respectively, such that $F V(y)$ is a homeomorphism from $V(y)$ onto $U(F y)$. For local homeomorphisms, Theorem 2.8 can be strengthened, and it turns out that in this case the sets $Q_{\mu}$ occurring in that theorem are the $\phi\left[\mathscr{P}_{X}\right]$-path components of $Y$.

3.2. Theorem. Suppose that for the local homeomorphism $F: Y \rightarrow X$ from $Y$ onto $X$, the inverse graph $\phi=\Gamma_{F}^{-1}$ has the path-lifting property for some admissible set $\mathscr{P}_{X} \subset \mathscr{P}(X)$, and that $X$ is $\mathscr{P}_{X}$-simply-connected and locally $\mathscr{P}_{X}$-path-connected. Then for each $\phi\left[\mathscr{P}_{X}\right]$-path component $Y_{\mu}$ of $Y, F \mid Y_{\mu}$ is a bijection, and hence a homeomorphism from $Y_{\mu}$ onto $X$.

Proof. Observe first that with $\mathscr{P}_{X}$ also $\mathscr{P}_{Y}=\phi\left[\mathscr{P}_{X}\right]$ is admissible and hence that the $Y_{\mu}$ are well defined. Moreover, from the definition of $\mathscr{P}_{Y}$ follows that

$$
F \mathscr{P}_{Y} \subset \mathscr{P}_{X}
$$


By 2.8 there exists a family of continuous maps $F_{\mu}: X \rightarrow Y$ such that $x \phi F_{\mu} x$ and $\phi[x]=\left\{F_{\mu} x\right\}_{\mu \in M}$ for each $x \in X$, and the sets $Q_{\mu}=F_{\mu} X$ are $\mathscr{P}_{Y}$-path-connected. Clearly then, $F F_{\mu} x=x$ for all $x \in X, \mu \in M$, and if $y \in Q_{\mu}$, then $y=F_{\mu} x$ for some $x \in X$, and because of $x \phi y$ we have $F y=x$ and thus $y=F_{\mu} F y$ for all $y \in Q_{\mu}$. This shows that $F \mid Q_{\mu}$ is a bijection from $Q_{\mu}$ onto $X$.

Note now that $Q_{\mu}$ is contained in some $\mathscr{P}_{Y}$-path component $Y_{\mu}$ of $Y$. Recall, moreover, from the proof of 2.8 that for a fixed $x_{0} \in X$ and $y_{\mu} \in \phi\left[x_{0}\right]$ we have $y \in Q_{\mu}$ if and only if there exist paths $p \in \mathscr{P}_{X}$ and $q \in \mathscr{P}(Y)$ such that $q(0)=y_{\mu}$, $q(1)=y$ and $p(t) \phi q(t), t \in J$. But if $y \in Y_{\mu}$ is any point, then $y_{\mu} \in Q_{\mu}$ implies that there is a path $q \in \mathscr{P}_{Y}$ connecting $y_{\mu}$ and $y$, and because of (4), $p(t)=F(q(t)), t \in J$, defines a path of $\mathscr{P}_{X}$ for which $p(t) \phi q(t), t \in J$. Thus $y \in Q_{\mu}$ and therefore $Q_{\mu}=Y_{\mu}$. Since $F_{\mu}=\left(F \mid Y_{\mu}\right)^{-1}$ is continuous, this completes the proof.

In many applications the following corollary will be useful:

3.3. Corollary. Let $F: Y \rightarrow X$ be a local homeomorphism from $Y$ onto $X$ and suppose that $Y$ is $\mathscr{P}(Y)$-path-connected and $X$ is $\mathscr{P}(X)$-simply-connected and locally $\mathscr{P}(X)$-path-connected. Then $F$ is a bijection, and hence a homeomorphism, from $Y$ onto $X$ if and only if $\Gamma_{F}^{-1}$ has the $\mathscr{P}(X)$-continuation property.

The necessity of the continuation property added in this result is self-evident, and the sufficiency follows directly from 3.2 since the obvious relations $\phi[\mathscr{P}(X)]$ $\subset \mathscr{P}(Y)$ and $F \mathscr{P}(Y) \subset \mathscr{P}(X)$ together imply that $\mathscr{P}(Y)=\phi[\mathscr{P}(X)]$.

For the case of mappings between Hausdorff topological linear spaces we can combine this result with Theorem 2.11:

3.4. TheOREM. Let $F: D \subset W \rightarrow V$ be a local homeomorphism from the $\mathscr{P}(W)$ path-connected subset $D$ of the Hausdorff topological linear space $W$ into another such space $V$, and suppose that $F(D)$ is open. Then $F$ is a bijection, and hence a homeomorphism, from $D$ onto $V$ if and only if $\phi=\Gamma_{F}^{-1}$ has the $\mathscr{P}(V)$-continuation property.

The necessity is again evident. Since $D(\phi)=F(D)$ is open and $V$ is $\mathscr{P}(V)$-pathconnected it follows from 2.11 that $F(D)=V$. But then the remainder of the statement is a direct consequence of 3.3 since $V$ is certainly $\mathscr{P}(V)$-simply-connected and locally $\mathscr{P}(V)$-path-connected.

The continuation property is an operational condition that may be difficult to verify in concrete situations. A rather simple case in which this property is easily established is contained in the following result:

3.5. THEOREM. Let $F: W \rightarrow V$ be a continuous map from the Banach space $W$ into the normed linear space $V$ such that $F(W)$ is open in $V$ and that

$$
\left\|F y_{1}-F y_{2}\right\| \geqq \gamma\left\|y_{1}-y_{2}\right\|, \quad y_{1}, y_{2} \in W, \quad \gamma>0 .
$$

Then $F$ is a bijection, and hence a homeomorphism, from $W$ onto $V$.

Proof. Clearly, $F$ is injective and $F^{-1}$ is continuous. Thus $\phi=\Gamma_{F}^{-1}$ is a local mapping relation with $D(\phi)=F(W)$. Let $p \in \mathscr{P}(V)$ be any path and suppose that 
$q:[0, \hat{t}) \subset J \rightarrow W$ is a continuous function for which $F q(t)=p(t)$ for $0 \leqq t<\hat{t}$. Then for any sequence $\left\{t_{k}\right\} \subset[0, \hat{t})$ with $\lim _{k \rightarrow \infty} t_{k}=\hat{t}$ we have

$$
\left\|p\left(t_{k}\right)-p\left(t_{j}\right)\right\|=\left\|F q\left(t_{k}\right)-F q\left(t_{j}\right)\right\| \geqq \gamma\left\|q\left(t_{k}\right)-q\left(t_{j}\right)\right\|
$$

which shows that $\left\{q\left(t_{k}\right)\right\}$ is a Cauchy sequence and hence convergent. If $\lim _{k \rightarrow \infty} q\left(t_{k}\right)$ $=\hat{y}$ then by continuity $F \hat{y}=p(\hat{t})$ and therefore $\phi$ has the continuation property for $\mathscr{P}(V)$ and the statement follows from 2.11.

This result is related to one of the main theorems in the theory of monotone mappings which states that a continuous, strongly monotone mapping from a Hilbert space into itself is bijective (see, e.g., Browder [1] and Minty [11]). In fact, under these conditions the mapping is open (see Schwartz [13]), and the strong monotonicity implies that (5) holds.

In the theory of monotone mappings, a so-called coerciveness condition plays an important role. The following definition introduces a modification of that condition:

3.6. Definition. A mapping $F: D \subset V \rightarrow V$ with domain and range in a normed linear space $V$ is called norm-coercive if for any $\gamma \geqq 0$ there exists a closed, bounded set $D_{\gamma} \subset D$ such that $\|F y\|>\gamma$ for all $y \in D \sim D_{\gamma}$.

Note that for $D=V, F$ is norm-coercive if and only if $\|F y\| \rightarrow \infty$ as $\|y\| \rightarrow \infty$. Observe also that (5) implies norm-coerciveness on $W$.

3.7. THEOREM. Let $V$ be a normed linear space and $G: D \subset V \rightarrow V$ a sequentially compact mapping on the $\mathscr{P}(V)$-path-connected set $D \subset V$. Suppose further that $F=I-G$ is a norm-coercive local homeomorphism and that $F(D)$ is open. Then $F$ is a bijection, and hence a homeomorphism, from $D$ onto $V$.

Proof. If we can show that $\phi=\Gamma_{F}^{-1}$ has the $\mathscr{P}(V)$ continuation property, then the result follows from 3.4. Let therefore $p \in \mathscr{P}(V)$ be given and $q:[0, \hat{t}) \subset J \rightarrow D$ a continuous function with $F q(t)=p(t)$ for $0 \leqq t<\hat{t}$. Since $p(J)$ is compact, $\gamma=$ $\max _{t \in J} p(t)<+\infty$ and by norm-coerciveness there is a closed bounded set $D_{\gamma} \subset D$ such that $\|F x\|>\gamma$ for all $x \in D \sim D_{\gamma}$. But then necessarily $q(t) \in D_{\gamma}$ for $t \in[0, \hat{t})$. Hence if $\left\{t_{k}\right\} \subset[0, \hat{t})$ is of any sequence with $\lim _{k \rightarrow \infty} t_{k}=\hat{t}$, then $\left\{G q\left(t_{k}\right)\right\} \subset G D_{y}$ has a convergent subsequence. Suppose this subsequence is again denoted by $\left\{G q\left(t_{k}\right)\right\}$ and that $\lim _{k \rightarrow \infty} G q\left(t_{k}\right)=\hat{y}^{\prime}$. If $\lim _{k \rightarrow \infty} F q\left(t_{k}\right)=\lim _{k \rightarrow \infty} p\left(t_{k}\right)=p(\hat{t})=\hat{y}$, then

$$
\begin{aligned}
\left\|q\left(t_{k}\right)-\left(\hat{y}+\hat{y}^{\prime}\right)\right\| & <\left\|q\left(t_{k}\right)-G q\left(t_{k}\right)-\hat{y}\right\|+\left\|G q\left(t_{k}\right)-\hat{y}^{\prime}\right\| \\
& =\left\|F q\left(t_{k}\right)-\hat{y}\right\|+\left\|G q\left(t_{k}\right)-\hat{y}^{\prime}\right\|
\end{aligned}
$$

and hence $\lim _{k \rightarrow \infty} q\left(t_{k}\right)=\hat{y}+\hat{y}^{\prime}$. Because $\left\{q\left(t_{k}\right)\right\} \subset D_{\gamma}$ and $D_{\gamma}$ is closed, we have $\hat{y}+\hat{y}^{\prime} \in D_{\gamma} \subset D$ and hence by continuity $F\left(\hat{y}+\hat{y}^{\prime}\right)=p(\hat{t})$ which proves that $\Gamma_{F}^{-1}$ indeed has the $\mathscr{P}(V)$ continuation property.

Although our definition of norm-coerciveness covers the coerciveness condition used in the theory of monotone mappings, this theorem does not include the corresponding theorem for monotone maps. But it supports again the frequently- 
stated observation that monotone mappings have many of the properties of compact operators.

For finite dimensional spaces Theorem 3.7 can, of course, be simplified considerably.

3.8. TheOREM. Let $V$ be a finite dimensional normed linear space, and $F: D \subset V$ $\rightarrow V$ a local homeomorphism on the $\mathscr{P}(V)$-path-connected set $D$ such that $F(D)$ is open. Then $F$ is a bijection, and hence a homeomorphism, from $D$ onto $V$ if and only if $F$ is norm-coercive.

The necessity added here can be proved as follows. If $F$ is a homeomorphism from $D$ onto $V$ then for any $\gamma \geqq 0, D_{\gamma}=F^{-1} S$ with $S=\bar{S}(0, \gamma)=\{x \in V \mid\|x\| \leqq \gamma\}$ is closed and bounded. Hence $y \in D \sim D_{\gamma}$ implies that $F y \notin S$ or $\|F y\|>\gamma$, and thus that $F$ is norm-coercive.

The local homeomorphism condition in the last theorems can, of course, be replaced by assumptions guaranteeing the validity of some local inverse function theorem. For instance, let us consider the following version of a well-known theorem of Hildebrandt and Graves [8]:

For the mapping $F: D \subset W \rightarrow V$ between the Banach spaces $W, V$ suppose there exists a linear operator $A: W \rightarrow V$ with bounded inverse $A^{-1} \in L(V, W)$, such that

$$
\left\|F y_{2}-F y_{1}-A\left(y_{2}-y_{1}\right)\right\| \leqq \alpha\left\|y_{2}-y_{1}\right\|<\left\|A^{-1}\right\|^{-1}\left\|y_{2}-y_{1}\right\|
$$

for all $y_{1}, y_{2}$ from some ball $\bar{S}\left(y_{0}, \rho\right) \subset D$. Let $\sigma=\left(\left\|A^{-1}\right\|^{-1}-\alpha\right) \rho$ and $U=$ $F^{(-1)}\left(\bar{S}\left(F y_{0}, \sigma\right)\right) \cap \bar{S}\left(y_{0}, \rho\right)$ then $F \mid U$ is a homeomorphism from $U$ onto $\bar{S}\left(F y_{0}, \sigma\right)$.

The proof is well known.

In order to apply our previous theorems we need to establish conditions which assure the continuation property for $\Gamma_{F}^{-1}$. A rather simple approach along this line leads to the following theorem essentially due to Ehrmann [5]:

3.9. TheOREM. Let $F: D \subset W \rightarrow V$ be a continuous mapping from the open, $\mathscr{P}(W)$-path-connected subset $D$ of the Banach space $W$ into the Banach space $V$. Suppose that for each $y \in D$ there exists a linear operator $A_{y}: W \rightarrow V$ with bounded inverse $A_{y}^{-1} \in L(V, W)$ such that (6) holds for all $y_{1}, y_{2}$ from some closed ball $\bar{S}\left(y, \rho_{y}\right) \in D$ (with $A$ and $\alpha$ replaced by $A_{y}$ and $\alpha_{y}$, respectively). If $\sigma_{y}=$ $\left(\left\|A_{y}^{-1}\right\|^{-1}-\alpha_{y}\right) \rho_{y} \geqq \sigma>0$ for $y \in D$, then $F$ is a homeomorphism from $D$ onto $V$.

From the inverse function theorem it follows that $F$ is a local homeomorphism, and since $\sigma>0$ is independent of $y \in D$, it is easily verified that $\Gamma_{F}^{-1}$ is a covering relation. Thus, by $2.10, \Gamma_{F}^{-1}$ has the path-lifting property and hence the result is a direct consequence of 3.4 .

Instead of the assumption that $\sigma$ is independent of $y$, we can, of course, also use the norm-coerciveness as a tool for assuring the continuation property. For example, by using the inverse function theorem in its conventional form for continuously (Fréchet)-differentiable operators, (i.e., by setting $A=F^{\prime}(y)$ in the above version), we obtain the following corollary of 3.8 . 
3.10. Corollary. Let $V$ be a finite dimensional normed linear space and suppose the mapping $F: D \subset V \rightarrow V$ has a continuous, nonsingular derivative on the open set $D \subset V$. If $F$ is norm-coercive on $D$, then for each $\mathscr{P}(V)$-path component $D_{\mu}$ of $D$, $F \mid D_{\mu}$ is a homeomorphism from $D_{\mu}$ onto $V$.

We conclude this section by showing that also the following well-known theorem of Hadamard [7] and Levy [9] (see, e.g., Schwartz [13]) can be proved by means of our results.

3.11. Theorem. Let $F: W \rightarrow V$ be a continuous (Fréchet)-differentiable map from the Banach space $W$ into the Banach space $V$, and suppose that for all $y \in W$, $F^{\prime}(y)$ has a bounded inverse $\left(F^{\prime}(y)\right)^{-1} \in L(V, W)$ such that $\left\|\left(F^{\prime}(y)\right)^{-1}\right\| \leqq \gamma$. Then, $F$ is a homeomorphism, and hence a diffeomorphism, from $W$ onto $V$.

Proof. By the standard inverse function theorem we know that $F$ is a local homeomorphism from $W$ onto the open subset $F(W)$ of $V$. Moreover, $F^{-1}$ is again continuously differentiable and we have for all $y \in W$

$$
\left(F^{-1}(F y)\right)^{\prime}=\left(F^{\prime}(y)\right)^{-1}
$$

Let $\mathscr{P}_{W}^{\prime} \subset \mathscr{P}(W)$ and $\mathscr{P}_{V}^{\prime} \subset \mathscr{P}(V)$ be the classes of all piecewise continuously differentiable paths in $W$ and $V$, respectively. Clearly then, $F \mathscr{P}_{W}^{\prime} \subset \mathscr{P}_{V}^{\prime}$. Conversely, if $F q(t)=p(t)$ for $t \in J$, i.e., if $q \in \mathscr{P}(W)$ is a $\Gamma_{F}^{-1}$-lifting of $p \in \mathscr{P}_{V}^{\prime}$, then clearly also $q$ is again piecewise continuously differentiable and because of (7) we have in each continuity interval of $p$

$$
q^{\prime}(t)=\left[F^{\prime}(q(t))\right]^{-1} p^{\prime}(t) .
$$

Hence, we have $q \in \mathscr{P}_{W}$ and, therefore, altogether $\phi\left[\mathscr{P}_{V}^{\prime}\right]=\mathscr{P}_{W}^{\prime}$. In order to show that $\Gamma_{F}^{-1}$ has the continuation property for $\mathscr{P}_{V}^{\prime}$ let $p \in \mathscr{P}_{V}^{\prime}$ and $q:[0, \hat{t}) \subset J \rightarrow W$ be a continuous function with $F q(t)=p(t)$ for $0 \leqq t<\hat{t}$. Then, by the same argument as above, $q$ is piecewise continuously differentiable on $[0, \hat{t})$ and $(8)$ holds on the continuity intervals of $p$. Let $\left\{t_{k}\right\} \subset[0, \hat{t})$ be any increasing sequence with $\lim _{k \rightarrow \infty} t_{k}=\hat{t}$. Then we have for $k<j$

$$
\begin{aligned}
\left\|q\left(t_{j}\right)-q\left(t_{k}\right)\right\| & \leqq \int_{t_{k}}^{t_{j}}\left\|q^{\prime}(s)\right\| d s \\
& \leqq \int_{t_{k}}^{t_{j}}\left\|\left[F^{\prime}(q(s))\right]^{-1}\right\|\left\|p^{\prime}(s)\right\| d s \leqq \gamma \max _{s \in J}\left\|p^{\prime}(s)\right\|\left|t_{j}-t_{k}\right|
\end{aligned}
$$

which shows that $\left\{q\left(t_{k}\right)\right\}$ is a Cauchy sequence and hence convergent. Thus, because of the continuity of $F$ on $W$ it follows immediately that $\Gamma_{F}^{-1}$ has the continuation property for $\mathscr{P}_{V}^{\prime}$. Since $F(W)$ is open in $V, 2.11$ therefore implies that $F(W)=V$, and now the result follows directly from 3.2.

It appears to be possible to extend this theorem to more general spaces using the tools developed here. We shall not enter into this in this paper. 
Recently, Meyer [10] extended 3.11 to the case where $\left\|\left(F^{\prime}(y)\right)^{-1}\right\|$ is allowed to go to infinity at most linearly in $\|y\|$. The following theorem is a modification of Meyer's result.

3.12. THEOREM. Let $G: V \rightarrow V$ be compact and continuously differentiable on the Banach space $V$. Set $F=I-G$ and suppose that $F^{\prime}(y)$ has a bounded inverse $\left(F^{\prime}(y)\right)^{-1} \in L(V, V)$ such that $\left\|\left(F^{\prime}(y)\right)^{-1}\right\| \leqq \alpha\|y\|+\beta$ for all $y \in V$. Then, $F$ is a homeomorphism, and hence a diffeomorphism, from $V$ onto itself.

Proof. We use the same notation as in the proof of 3.11 of course with $W=V$. Let $p \in \mathscr{P}_{V}^{\prime}$ and let $q:[0, \hat{t}) \subset J \rightarrow V$ be a continuous function such that $F q(t)=p(t)$ for $0 \leqq t<\hat{t}$. Then, as in the case of (9), we find that

$$
\begin{aligned}
\|q(t)-q(0)\| & \leqq \int_{0}^{t}\left\|q^{\prime}(s)\right\| d s \leqq \int_{0}^{t}\left\|\left(F^{\prime}(q(s))\right)^{-1}\right\|\left\|p^{\prime}(s)\right\| d s \\
& \leqq \hat{\beta} t+\int_{0}^{t} \hat{\alpha}\|q(s)-q(0)\| d s
\end{aligned}
$$

where

$$
\hat{\beta}=(\alpha\|q(0)\|+\beta) \max _{s \in J}\left\|p^{\prime}(s)\right\|, \quad \hat{\alpha}=\alpha \max _{s \in J}\left\|p^{\prime}(s)\right\| .
$$

The well-known Gronwall inequality now implies that

$$
\|q(t)-q(0)\| \leqq \hat{\beta}\left(e^{\hat{\alpha} \hat{t}}-1\right) / \hat{\alpha}, \quad t \in[0, \hat{t}),
$$

i.e., for any sequence $\left\{t_{k}\right\} \subset[0, \hat{t})$ with $\lim _{k \rightarrow \infty} t_{k}=\hat{t}$ it follows that $\left\{q\left(t_{k}\right)\right\}$ is bounded. Because of the compactness of $G$, there exists a convergent subsequence of $\left\{G q\left(t_{k}\right)\right\}$. If this subsequence is again denoted by $\left\{G q\left(t_{k}\right)\right\}$ and if $\lim _{k \rightarrow \infty} G q\left(t_{k}\right)=\hat{y}^{\prime}$, then with $\lim _{k \rightarrow \infty} F q\left(t_{k}\right)=\lim _{k \rightarrow \infty} p\left(t_{k}\right)=\hat{y}$ it follows that

$$
\left\|q\left(t_{k}\right)-\left(\hat{y}+\hat{y}^{\prime}\right)\right\| \leqq\left\|q\left(t_{k}\right)-G q\left(t_{k}\right)-\hat{y}\right\|+\left\|G q\left(t_{k}\right)-\hat{y}^{\prime}\right\|
$$

which proves that $\Gamma_{F}^{-1}$ has the continuation property for $\mathscr{P}_{V}^{\prime}$. The remainder of the proof is identical to that of 3.11 .

4. Applications to equations $F(x, y)=0$. In this section we shall discuss a few possible results about equations of the form $F(x, y)=0$ where $F: D \subset X \times Y \rightarrow Z$ and $X, Y, Z$ are Banach spaces. The relation to be considered is now

$$
\phi=\{(x, y) \in D \mid F(x, y)=0\} .
$$

There are various possibilities of assuring that $\phi$ has the local mapping property. We shall restrict ourselves to those derivable from the classical implicit function theorem, and use that theorem in the following form:

Let $F: D \subset X \times Y \rightarrow Z$ be continuous on $D$ and $F\left(x_{0}, y_{0}\right)=0$ at $\left(x_{0}, y_{0}\right) \in D$ with $\bar{S}\left(x_{0}, \sigma\right) \times \bar{S}\left(y_{0}, \rho_{0}\right) \subset D$. Suppose $A: Y \rightarrow Z$ is a linear operator with bounded inverse $A^{-1} \in L(Z, Y)$ such that

$$
\left\|F\left(x, y_{2}\right)-F\left(x, y_{1}\right)-A\left(y_{2}-y_{1}\right)\right\| \leqq \alpha_{0}\left\|y_{2}-y_{1}\right\|<\left\|A^{-1}\right\|^{-1}\left\|y_{2}-y_{1}\right\|
$$


for $x \in \bar{S}\left(x_{0}, \sigma\right)$ and $y_{1}, y_{2} \in \bar{S}\left(y_{0}, \rho_{0}\right)$. Finally, let $\sigma_{0} \leqq \sigma$ be such that $\left\|F\left(x, y_{0}\right)\right\|$ $\leqq\left(\left\|A^{-1}\right\|^{-1}-\alpha_{0}\right) \rho_{0}$ for $x \in \bar{S}\left(x_{0}, \sigma_{0}\right)$. Then for each $x \in \bar{S}\left(x_{0}, \sigma_{0}\right)$ the equation $F(x, y)=0$ has a solution $y_{x} \in \bar{S}\left(y_{0}, \rho_{0}\right)$ which is unique in that ball and depends continuously on $x$.

The proof is standard and is omitted here (see, e.g., Hildebrandt and Graves [8], and Cesari [2]).

Our formulation does not represent the weakest possible result. In fact, in going through the proof it is readily seen that the continuity assumption about $F$ can be reduced to separate continuity in each variable. Also, the conditions on $A$ can be weakened, as Cesari has shown. However, the conventional implicit function theorem for continuously differentiable operators is contained in this particular version.

Based on this implicit function theorem, we obtain the following result assuring that $\phi$ is a local mapping relation:

4.1. Lemma. Let $F: D \subset X \times Y \rightarrow Z$ be continuous on the open set $D$ in $X \times Y$, and suppose that for each $(x, y) \in D$ there exists a linear operator $A(x, y): Y \rightarrow Z$ with bounded inverse $A^{-1}(x, y) \in L(Z, Y)$ such that $A$ depends continuously on $(x, y)$ in the strong operator topology. Finally, assume there exists an $\alpha>0$ such that for each $(x, y) \in D a \rho=\rho(x, y)>0$ can be found with

$$
\left\|F\left(x, y_{2}\right)-F\left(x, y_{1}\right)-A(x, y)\left(y_{2}-y_{1}\right)\right\| \leqq \alpha\left\|y_{2}-y_{1}\right\|<\left\|A^{-1}(x, y)\right\|^{-1}\left\|y_{2}-y_{1}\right\|
$$

for $y_{1}, y_{2} \in \bar{S}(y, \rho)$. Then $\phi$ is a local mapping relation and $D(\phi)$ is open in $X$.

Proof. For $\left(x_{0}, y_{0}\right) \in \phi$ we can find $\sigma>0, \rho>0$ such that $\bar{S}\left(x_{0}, \sigma\right) \times \bar{S}\left(y_{0}, \rho\right) \subset D$. Set $A \equiv A\left(x_{0}, y_{0}\right)$ and let $\varepsilon>0$ be such that $\alpha_{0}=\alpha+\varepsilon\left\|A^{-1}\right\|^{-1}$. Then, $\sigma_{0} \in(0, \sigma]$, $\rho_{0} \in\left(0, \rho\left(x_{0}, y_{0}\right)\right]$ can be chosen such that $\left\|A(x, y)-A\left(x_{0}, y_{0}\right)\right\| \leqq \varepsilon$ and $\left\|F\left(x, y_{0}\right)\right\|$ $<\left(\left\|A^{-1}\right\|^{-1}-\alpha_{0}\right) \rho_{0}$ for $x \in \bar{S}\left(x_{0}, \sigma_{0}\right)$ and $y \in \bar{S}\left(y_{0}, \rho_{0}\right)$. Then for any $x \in \bar{S}\left(x_{0}, \sigma_{0}\right)$ and $y_{1}, y_{2} \in \bar{S}\left(y_{0}, \rho_{0}\right)$

$$
\begin{aligned}
\left\|F\left(x, y_{2}\right)-F\left(x, y_{1}\right)-A\left(x_{0}, y_{0}\right)\left(y_{2}-y_{1}\right)\right\| \leqq & \left\|F\left(x, y_{2}\right)-F\left(x, y_{1}\right)-A\left(x, y_{1}\right)\left(y_{2}-y_{2}\right)\right\| \\
& +\left\|A\left(x, y_{1}\right)-A\left(x_{0}, y_{0}\right)\right\|\left\|y_{2}-y_{1}\right\| \\
& \leqq \\
& (\alpha+\varepsilon)\left\|y_{2}-y_{1}\right\| .
\end{aligned}
$$

Thus, all conditions of the implicit function theorem are satisfied and hence the restriction $\varphi=\phi \cap\left(S\left(x_{0}, \sigma_{0}\right) \times X\left(y_{0}, \rho_{0}\right)\right)$ is a continuous map from $S\left(x_{0}, \sigma_{0}\right)$ into $S\left(y_{0}, \rho_{0}\right)$. Since $\left(x_{0}, y_{0}\right) \in \phi$ was arbitrary, this completes the proof.

Condition (7) is evidently satisfied if $F$ has a continuous partial derivative $\partial_{2} F(x, y)$ in $D$ with a bounded inverse $\left(\partial_{2} F(x, y)\right)^{-1} \in L(Z, Y)$.

The question now arises when $\phi$ has the path-lifting property. As in $\S 3$, a possible approach here is to add conditions to 4.1 which assure that $\phi$ is a covering relation. Loosely speaking, these conditions have to guarantee that the radius $\sigma_{0}$ does not depend on $\left(x_{0}, y_{0}\right) \in \phi$. This means, of course, that we have to make uniformity 
assumptions about the continuity of $A, F$, etc. One possible result along this line can be stated as follows:

4.2. TheOREM. Let $F: D \subset X \times Y \rightarrow Z$ be uniformly continuous on the open set $D$ in $X \times Y$, and suppose that for each $(x, y) \in D$ a linear operator $A(x, y): Y \rightarrow Z$ with bounded inverse $A^{-1}(x, y) \in L(Z, Y)$ is defined such that $\left\|A^{-1}(x, y)\right\|^{-1} \geqq \mu>0$, for all $(x, y) \subset D$. Assume further that $A$ is uniformly continuous for $(x, y) \in D$. Finally, let $\alpha>0$ and $\rho>0$ be such that for each $(x, y) \in D$ the inequality (7) holds with $\alpha<\mu$ for all $y_{1}, y_{2} \in \bar{S}(y, \rho)$. Then, $\phi$ is a covering relation.

Proof. By going through the proof of 4.1 again, we see that both $\rho_{0}$ and $\sigma_{0}$ can now be chosen independently of $\left(x_{0}, y_{0}\right) \in \phi$. Hence, the restriction $\varphi$ is a continuous mapping from $S\left(x_{0}, \sigma_{0}\right)$ into $S\left(y_{0}, \rho_{0}\right)$ and for every $x \in S\left(y_{0}, \sigma_{0}\right)$, $\varphi(x) \in S\left(y_{0}, \rho_{0}\right)$ is the only solution of $F(x, y)=0$ in that ball. Clearly then, $\phi\left[S\left(x_{0}, \sigma_{0}\right)\right] \subset \bigcup_{y_{0} \in \phi\left[x_{0}\right]}\left(S\left(y_{0}, \rho_{0}\right) \cap R(\phi)\right)$ and the sets on the right are disjoint and open in $R(\phi)$. This proves that $\phi$ is indeed a covering relation.

Theorem 4.2 essentially generalizes the first main theorem of Ficken [6] who considers an operator $F: J \times D_{Y} \subset R^{1} \times Y \rightarrow Z$ and uses instead of the operator function $A$ the partial derivative $\partial_{2} F(s, y)$, yet who places slightly less stringent continuity assumptions upon $F$. As indicated earlier, our continuity assumptions for $F$ in the basic implicit function theorem, and therefore also in 4.2, can also be weakened to correspond to those of Fricken.

As in $\S 3$ we can also assure the path-lifting property for $\phi$ by making assumptions about $R(\phi)$. Following is a simple result along this line:

4.3. Theorem. Suppose that $F: D \subset X \times Y \rightarrow Z$ is continuous on $D$, and that $\phi$ has the local mapping property. If $R(\phi)$ is compact, then $\phi$ has the $\mathscr{P}(D(\phi))$ continuation property.

Proof. Let $p \in \mathscr{P}(D(\phi))$ and $q:[0, \hat{t}) \subset J \rightarrow R(\phi)$ be a continuous map such that $F(p(t), q(t))=0$ for $0 \leqq t<\hat{t}$. If $\left\{t_{k}\right\} \subset[0, \hat{t})$ is any sequence of $\lim _{k \rightarrow \infty} t_{k}=\hat{t}$, then $\left\{q\left(t_{k}\right)\right\} \subset R(\phi)$ and hence there exists a convergent subsequence. For the sake of simplicity assume that $\lim _{k \rightarrow \infty} q\left(t_{k}\right)=\hat{y} \in R(\phi)$. Since $p(\hat{t}) \in D(\phi)$, we have $(p(\hat{t}), \hat{y})$ $\in D(\phi) \times R(\phi) \subset D$ and, therefore, by the continuity of $F, F(p(\hat{t}), \hat{y})=0$, i.e., $\phi$ has the continuation property for $\mathscr{P}(D(\phi))$.

In particular, note that under the conditions of $4.3, D(\phi)$ is $\mathscr{P}(X)$-simply connected and locally $\mathscr{P}(X)$-path-connected and if $F(x, y)$ has for one $x \in D(\phi)$ only one solution $y \in R(\phi)$ then by 2.8 there exists a continuous function $G: D(\phi)$ $\rightarrow R(\phi)$ such that $F(x, G x)=0$ for all $x \in D(\phi)$ and, moreover, $y=G x$ is the only solution of $F(x, y)=0$ in $R(\phi)$.

This result, together with 4.1 , represents essentially an extension of the second main result of Ficken [6], except for the form of the continuity assumption about $F$. In this case this continuity assumption can again be weakened if the local mapping property is derived from conditions of the type used in 4.2. The compactness 
assumptions about $R(\phi)$ can be guaranteed by suitable compactness conditions about $F$. For both these points we refer to Ficken [6].

\section{REFERENCES}

1. F. E. Browder, The solvability of non-linear functional equations, Duke Math. J. 30 (1963), 557-566.

2. L. Cesari, The implicit function theorem in functional analysis, Duke Math. J. 33 (1966), 417-440.

3. D. F. Davidenko, An application of the method of variation of parameters to the construction of iterative formulas of increased accuracy for numerical solutions of nonlinear equations, Dokl. Akad. Nauk SSSR 162 (1965), 702-706=Soviet Math. Dokl. 6 (1965), 702-706.

4. J. Davis, The solution of nonlinear operator equations with critical points, Tech. Rep. 25, Dept. of Math., Oregon State Univ., Corvallis, 1966.

5. H. Ehrmann, On implicit function theorems and the existence of solutions of nonlinear equations, Enseignement Math. (2) 9 (1963), 129-176.

6. F. A. Ficken, The continuation method for functional equations, Comm. Pure Appl. Math. 4 (1951), 435-456.

7. J. Hadamard, Sur les transformations ponctuelles, Bull. Soc. Math. France 34 (1906), 71-84.

8. T. H. Hildebrandt and L. M. Graves, Implicit functions and their differentials in general analysis, Trans. Amer. Math. Soc. 29 (1927), 127-153.

9. M. P. Levy, Sur les fonctions de lignes implicites, Bull. Soc. Math. France 48 (1920), 13-27.

10. G. H. Meyer, On solving nonlinear equations with a one-parameter operator imbedding, Tech. Rep. 67-50, Comp. Science Center, Univ. of Maryland, College Park, 1967; SIAM J. Numer. Anal. (to appear).

11. G. J. Minty, Monotone (nonlinear) operators in Hilbert space, Duke Math. J. 29 (1962), 341-346.

12. H. Schubert, Topologie, Teubner, Stuttgart, Germany, 1964.

13. J. T. Schwartz, Nonlinear functional analysis (1963-1964), Lecture notes, Courant Inst. of Math. Sciences, New York Univ., 1965.

14. M. N. Yakovlev, On the solution of systems of nonlinear equations by differentiation with respect to a parameter, U.S.S.R. Comput. Math. and Math. Phys. 4 (1964), 146-149.

UNIVERSITY OF MARYLAND,

College Park, Maryland 